\title{
DETERMINING THE HUBBLE CONSTANT FROM HUBBLE PARAMETER MEASUREMENTS
}

\author{
Yun Chen $^{1}$, Suresh Kumar ${ }^{2}$, and Bharat Ratra ${ }^{3}$ \\ ${ }^{1}$ Key Laboratory for Computational Astrophysics, National Astronomical Observatories, Chinese Academy of Sciences, Beijing, 100012, China; chenyun @bao.ac.cn \\ ${ }^{2}$ Department of Mathematics, BITS Pilani, Pilani Campus, Rajasthan-333031, India; suresh.kumar@pilani.bits-pilani.ac.in \\ ${ }^{3}$ Department of Physics, Kansas State University, 116 Cardwell Hall, Manhattan, KS 66506, USA; ratra@ phys.ksu.edu \\ Received 2016 June 28; revised 2016 September 16; accepted 2016 December 7; published 2017 January 20
}

\section{ABSTRACT}

We use 28 measurements of the Hubble parameter, $H(z)$, at intermediate redshifts $0.07 \leqslant z \leqslant 2.3$ to determine the present-day Hubble constant $H_{0}$ in four cosmological models. We measure $H_{0}=68.3_{-2.6}^{+2.7}, 68.4_{-3.3}^{+2.9}, 65.0_{-6.6}^{+6.5}$, and $67.9_{-2.4}^{+2.4} \mathrm{~km} \mathrm{~s}^{-1} \mathrm{Mpc}^{-1}(1 \sigma$ errors) in the $\Lambda \mathrm{CDM}$ (spatially flat and non-flat), $\omega \mathrm{CDM}$, and $\phi \mathrm{CDM}$ models, respectively. These measured $H_{0}$ values are more consistent with the lower values determined from recent data on the cosmic microwave background and baryon acoustic oscillations, as well as with the value found from a median statistical analysis of Huchra's compilation of $H_{0}$ measurements, but include the higher local measurements of $H_{0}$ within the $2 \sigma$ confidence limits.

Key words: cosmological parameters - dark energy

\section{INTRODUCTION}

The current value of the cosmological expansion rate, the Hubble constant $H_{0}$, is an important cosmological datum. Although one of the most measured cosmological parameters, it was more than seven decades after Hubble's first measurement before a consensus value for $H_{0}$ started to emerge. In 2001 Freedman et al. (2001) provided $H_{0}=72 \pm$ $8 \mathrm{~km} \mathrm{~s}^{-1} \mathrm{Mpc}^{-1}$ ( $1 \sigma$ error including systematics) as a reasonable summary of the $H_{0}$ value from the Hubble Space Telescope Key Project. In the same year Gott et al. (2001) applied median statistics ${ }^{4}$ to $331 H_{0}$ estimates tabulated by Huchra $^{5}$ and determined $H_{0}=67 \pm 3.5 \mathrm{~km} \mathrm{~s}^{-1} \mathrm{Mpc}^{-1}$. During the following decade median statistics was applied to larger compilations of $H_{0}$ measurements from Huchra, in 2003 to 461 measurements by Chen et al. (2003), who found $H_{0}=68 \pm 3.5 \mathrm{~km} \mathrm{~s}^{-1} \mathrm{Mpc}^{-1}$, and in 2011 to 553 measurements by Chen \& Ratra (2011a), who found $H_{0}=68 \pm$ $2.8 \mathrm{~km} \mathrm{~s}^{-1} \mathrm{Mpc}^{-1}$.

Many more recent $H_{0}$ determinations are consistent with these results. For instance, the final Wilkinson Microwave Anisotropy Probe $(W M A P)$ measurement is $H_{0}=70.0 \pm 2.2$ $\mathrm{km} \mathrm{s}^{-1} \mathrm{Mpc}^{-1}$ (Hinshaw et al. 2013), while the Atacama Cosmology Telescope and the WMAP seven-year data on the anisotropy of the cosmic microwave background (CMB) give $H_{0}=70.0 \pm 2.4 \mathrm{~km} \mathrm{~s}^{-1} \mathrm{Mpc}^{-1}$ (Sievers et al. 2013); baryon acoustic oscillations (BAO), Type Ia supernovae, and CMB data result in $H_{0}=67.3 \pm 1.1 \mathrm{~km} \mathrm{~s}^{-1} \mathrm{Mpc}^{-1}$ (Aubourg et al. 2015; also see Ross et al. 2015; Bernal et al. 2016; L'Huillier \& Shafieloo 2016; Luković et al. 2016), with the Planck 2015 CMB data value being $H_{0}=67.8 \pm$ $0.9 \mathrm{~km} \mathrm{~s}^{-1} \mathrm{Mpc}^{-1}$ (Ade et al. 2015; but see Addison et al. 2016).

While the consistency of these results is encouraging, some recent local estimates of $H_{0}$ are larger. Riess et al. (2011) find

\footnotetext{
4 For applications and discussions of median statistics see Podariu et al. (2001), Chen \& Ratra (2003), Mamajek \& Hillenbrand (2008), Croft \& Dailey (2015), Andreon \& Hurn (2012), Farooq et al. (2013a), Crandall \& Ratra (2014, 2015), Ding et al. (2015), Crandall et al. (2015), and Zheng et al. (2016). Median statistics does not make use of the error bars of the individual mesaurements.

5 https://www.cfa.harvard.edu/ dfabricant/huchra/
}

$H_{0}=73.8 \pm 2.4 \mathrm{~km} \mathrm{~s}^{-1} \mathrm{Mpc}^{-1}$ (but see Efstathiou 2014, who argues that $H_{0}=72.5 \pm 2.5 \mathrm{~km} \mathrm{~s}^{-1} \mathrm{Mpc}^{-1}$ is a better representation), Freedman et al. (2012) find $H_{0}=74.3 \pm$ $2.1 \mathrm{~km} \mathrm{~s}^{-1} \mathrm{Mpc}^{-1}$, while Riess et al. (2016) give $H_{0}=73.24 \pm$ $1.74 \mathrm{~km} \mathrm{~s}^{-1} \mathrm{Mpc}^{-1}$.

It is important to understand the reasons for this difference. For instance, the value and uncertainty of $H_{0}$ affect observational constraints on other cosmological parameters (see, e.g., Samushia et al. 2007; Chen et al. 2016). Given current cosmological data, the standard model of particle physics with three light neutrino species is more compatible with the lower $H_{0}$ value and difficult to reconcile with the higher value (see, e.g., Calabrese et al. 2012); and the difference between the local and global $H_{0}$ values might be an indication that the $\Lambda \mathrm{CDM}$ model needs to be extended (see, e.g., Di Valentino et al. 2016).

Here we use measurements of the Hubble parameter, $H(z)$ (where $z$ is redshift), to determine the Hubble constant. $H(z)$ data have previously been used to constrain other cosmological parameters (see, e.g., Samushia \& Ratra 2006; Chen \& Ratra 2011b; Farooq et al. 2013b, 2015; Farooq \& Ratra 2013a; Capozziello et al. 2014; Chen et al. 2015; Meng et al. 2015; Alam et al. 2016; Guo \& Zhang 2016; Mukherjee 2016; Solà et al. 2016), including measuring the redshift of the cosmological deceleration-acceleration transition between the earlier nonrelativistic matter-dominated epoch and the current dark-energy-dominated epoch (see, e.g., Farooq \& Ratra 2013b; Moresco et al. 2016). See Verde et al. (2014) for an early attempt at measuring $H_{0}$ from $H(z)$ data. Here we use more data (28 versus 15 measurements) to higher redshift (2.30 versus 1.04) than Verde et al. (2014) used and so find tighter constraints on $H_{0}$.

We find that our $H_{0}$ values obtained from $H(z)$ are more consistent with the lower values determined using median statistics or from CMB anisotropy or BAO measurements, and with the predictions of the standard model of particle physics with only three light neutrino species and no "dark radiation." Systematic errors affecting $H(z)$ measurements are largely different from those affecting $\mathrm{CMB}$ and $\mathrm{BAO}$ measurements. In addition, median statistics does not make use of the error 
Table 1

Hubble Parameter vs. Redshift Data

\begin{tabular}{|c|c|c|c|}
\hline$z$ & $\begin{array}{c}H(z) \\
\left(\mathrm{km} \mathrm{s}^{-1} \mathrm{Mpc}^{-1}\right)\end{array}$ & $\left(\mathrm{km} \mathrm{s}^{-\sigma_{H}} \mathrm{Mpc}^{-1}\right)$ & Reference \\
\hline 0.070 & 69 & 19.6 & 5 \\
\hline 0.090 & 69 & 12 & 1 \\
\hline 0.120 & 68.6 & 26.2 & 5 \\
\hline 0.170 & 83 & 8 & 1 \\
\hline 0.179 & 75 & 4 & 3 \\
\hline 0.199 & 75 & 5 & 3 \\
\hline 0.200 & 72.9 & 29.6 & 5 \\
\hline 0.270 & 77 & 14 & 1 \\
\hline 0.280 & 88.8 & 36.6 & 5 \\
\hline 0.350 & 76.3 & 5.6 & 7 \\
\hline 0.352 & 83 & 14 & 3 \\
\hline 0.400 & 95 & 17 & 1 \\
\hline 0.440 & 82.6 & 7.8 & 6 \\
\hline 0.480 & 97 & 62 & 2 \\
\hline 0.593 & 104 & 13 & 3 \\
\hline 0.600 & 87.9 & 6.1 & 6 \\
\hline 0.680 & 92 & 8 & 3 \\
\hline 0.730 & 97.3 & 7.0 & 6 \\
\hline 0.781 & 105 & 12 & 3 \\
\hline 0.875 & 125 & 17 & 3 \\
\hline 0.880 & 90 & 40 & 2 \\
\hline 0.900 & 117 & 23 & 1 \\
\hline 1.037 & 154 & 20 & 3 \\
\hline 1.300 & 168 & 17 & 1 \\
\hline 1.430 & 177 & 18 & 1 \\
\hline 1.530 & 140 & 14 & 1 \\
\hline 1.750 & 202 & 40 & 1 \\
\hline 2.300 & 224 & 8 & 4 \\
\hline
\end{tabular}

References. (1) Simon et al. (2005), (2) Stern et al. (2010), (3) Moresco et al. (2012), (4) Busca et al. (2013), (5) Zhang et al. (2014), (6) Blake et al. (2012), (7) Chuang \& Wang (2013).

bars of the individual measurements. It is significant that all four techniques result in very similar values of $H_{0}$.

To determine $H_{0}$ we analyze the $H(z)$ data tabulated in Farooq \& Ratra (2013b) and reproduced in Table 1 here $^{6}$, using two different dark energy models, $\Lambda$ CDM (Peebles 1984) and $\phi$ CDM (Peebles \& Ratra 1988; Ratra \& Peebles 1988), as well as an incomplete, but popular, parameterization of dark energy, $\omega \mathrm{CDM}$. In all cases we measure $H_{0}$ from the one-dimensional likelihood determined by marginalizing over all other parameters. (Limits on other parameters, such as the current nonrelativistic matter density parameter, are quite reasonable.)

In the next section we summarize the models we use, as well as the $\omega \mathrm{CDM}$ parameterization. In Section 3 we present our $H_{0}$ determinations. We conclude in the final section.

\section{2. $\Lambda \mathrm{CDM}, \omega \mathrm{CDM}, \mathrm{AND} \phi \mathrm{CDM}$}

The Hubble parameter of the spatially flat $\Lambda$ CDM model is

$$
H(z)=H_{0} \sqrt{\Omega_{m 0}(1+z)^{3}+1-\Omega_{m 0}},
$$

\footnotetext{
6 The error bars of these $H(z)$ measurements include systematic errors. In the analyses here we ignore the correlations between the three points of Blake et al. (2012); these only very slightly affect the results (Farooq et al. 2016).
}

while in the general (non-flat) $\Lambda \mathrm{CDM}$ model it is

$$
\begin{aligned}
& H(z) \\
& =H_{0} \sqrt{\Omega_{m 0}(1+z)^{3}+\left(1-\Omega_{m 0}-\Omega_{\Lambda}\right)(1+z)^{2}+\Omega_{\Lambda}},
\end{aligned}
$$

where $\Omega_{m 0}$ is the current value of the nonrelativistic matter density parameter and $\Omega_{\Lambda}$ is the cosmological constant density parameter.

In the spatially flat $\omega \mathrm{CDM}$ parameterization we have

$$
H(z)=H_{0} \sqrt{\Omega_{m 0}(1+z)^{3}+\left(1-\Omega_{m 0}\right)(1+z)^{3\left(1+w_{X}\right)}},
$$

where $\omega_{X}$ is the constant, negative, equation-of-state parameter relating the (dark energy) $X$-fluid pressure and energy density through $p_{X}=\omega_{X} \rho_{X}$. The $\omega$ CDM parameterization is incomplete and does not consistently describe inhomogeneities. However, $\phi \mathrm{CDM}$, discussed next, is a consistent dynamical dark energy model.

The Friedmann equation of the spatially flat $\phi \mathrm{CDM}$ model is

$$
H^{2}(z)=\frac{8 \pi}{3 m_{p}^{2}}\left(\rho_{m}+\rho_{\phi}\right),
$$

where $m_{p}$ is the Planck mass, $\rho_{m}$ is the nonrelativistic matter energy density, and the energy density of the scalar field $\phi$ is

$$
\rho_{\phi}=\frac{m_{p}^{2}}{32 \pi}\left(\dot{\phi}^{2}+\kappa m_{p}^{2} \phi^{-\alpha}\right) .
$$

Here an overdot denotes a time derivative, $\kappa\left(m_{p}, \alpha\right)$ and $\alpha$ are positive constants, and we have picked an inverse-power-law potential energy density of the scalar field $V(\phi)=\kappa m_{p}^{2} \phi^{-\alpha} / 2$. The equation of motion of the scalar field is

$$
\ddot{\phi}+3 \frac{\dot{a}}{a} \dot{\phi}+\frac{d V}{d \phi}=0
$$

where $a$ is the scale factor. These equations are numerically integrated to provide $H(z)$ in the $\phi \mathrm{CDM}$ model (Peebles \& Ratra 1988; Samushia 2009; Farooq 2013).

\section{ANALYSIS AND RESULTS}

We constrain cosmological parameters by minimizing $\chi_{H}^{2}$ :

$$
\chi_{H}^{2}(\boldsymbol{p})=\sum_{i=1}^{N} \frac{\left[H^{\mathrm{th}}\left(z_{i} ; \boldsymbol{p}\right)-H^{\mathrm{obs}}\left(z_{i}\right)\right]^{2}}{\sigma_{H, i}^{2}},
$$

for $N$ measured values of $H^{\mathrm{obs}}\left(z_{i}\right)$ with variance $\sigma_{H, i}^{2}$ at redshift $z_{i}$ where $H^{\text {th }}$ is the predicted value of $H(z)$ in the cosmological model. $\boldsymbol{p}$ represents the free parameters of the cosmological model under consideration, $H_{0}$ and $\Omega_{m 0}$ in all four cases, with one additional parameter in three of the cases: $\Omega_{\Lambda}$ in non-flat $\Lambda \mathrm{CDM}, \omega_{X}$ in the spatially flat $\omega \mathrm{CDM}$ parameterization, and $\alpha$ in the spatially flat $\phi \mathrm{CDM}$ model. We use the compilation of $28 H(z)$ data points from Farooq \& Ratra (2013b) as reproduced here in Table 1 to constrain the model parameters under consideration by using the Markov chain Monte Carlo method coded in the publicly available package CosmoMC (Lewis \& Bridle 2002).

Our results are summarized in Table 2 and Figures 1-5.

The limits on cosmological parameters shown in Table 2 are derived from the corresponding one-dimensional likelihood 
Table 2

Mean Values of Free Parameters of Various Models with $1 \sigma$ and $2 \sigma$ Error Bars

\begin{tabular}{lcccc}
\hline \hline Parameter & $\Lambda$ CDM & Non-flat $\Lambda$ CDM & XCDM & $\phi$ CDM \\
\hline$H_{0}$ & $68.3_{-2.6-5.1}^{+2.7+5.2}$ & $68.4_{-3.3-5.4}^{+2.9+5.9}$ & $65.0_{-6.6-9.3}^{+6.5+9.4}$ & $67.9_{-2.4-4.7}^{+2.4+4.7}$ \\
$\Omega_{m 0}$ & $0.276_{-0.039-0.068}^{+0.032+0.072}$ & $0.267_{-0.050-0.102}^{+0.049+0.010}$ & $0.308_{-0.076-0.102}^{+0.048+0.114}$ & $0.275_{-0.035-0.062}^{+0.029+0.063}$ \\
$\Omega_{\Lambda}$ & $\cdots$ & $0.708_{-0.167-0.208}^{+0.101+0.219}$ & $\cdots$ & $\cdots$ \\
$w_{X}$ & $\cdots$ & $\cdots$ & $-0.780_{-0.292-0.414}^{+0.196+0.460}$ & $\cdots$ \\
$\alpha$ & $\cdots$ & $\cdots$ & $\cdots$ & no limits \\
\hline$\chi_{\min }^{2}$ & 17.0 & 16.9 & 17.0 & 17.0 \\
\hline
\end{tabular}

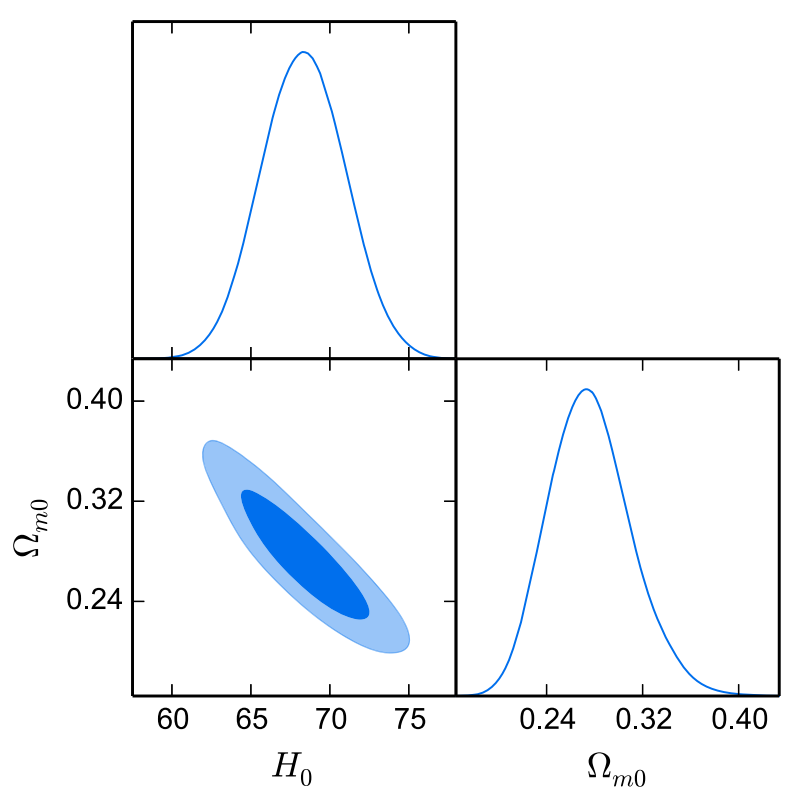

Figure 1. $1 \sigma$ and $2 \sigma$ confidence contours of the parameters of the spatially flat $\Lambda \mathrm{CDM}$ model. Marginalized probability distributions of the individual parameters are also displayed.

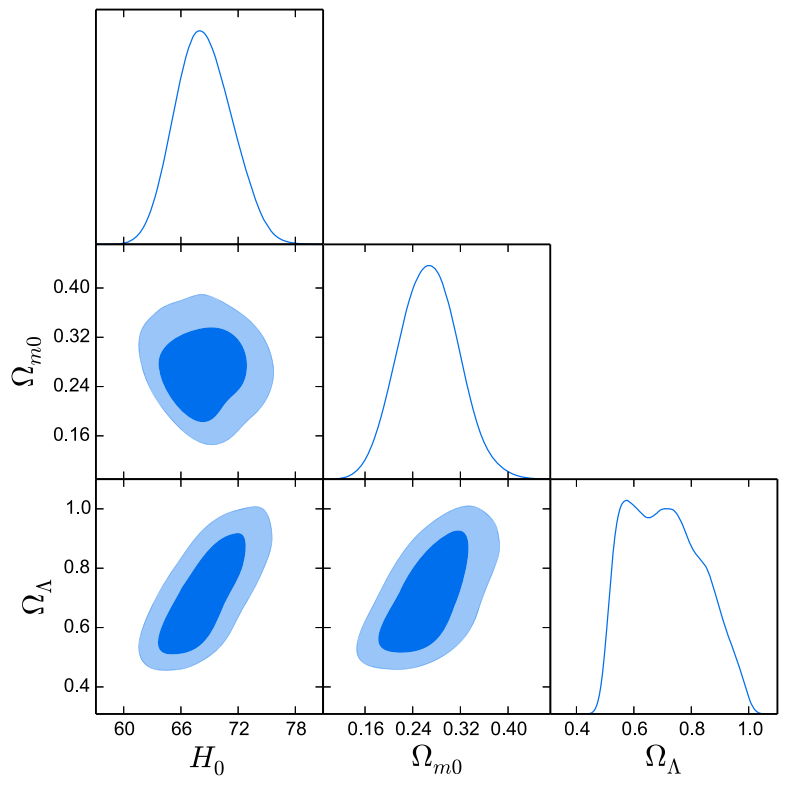

Figure 2. $1 \sigma$ and $2 \sigma$ confidence contours of the parameters of the non-flat $\Lambda \mathrm{CDM}$ model. Marginalized probability distributions of the individual parameters are also displayed.

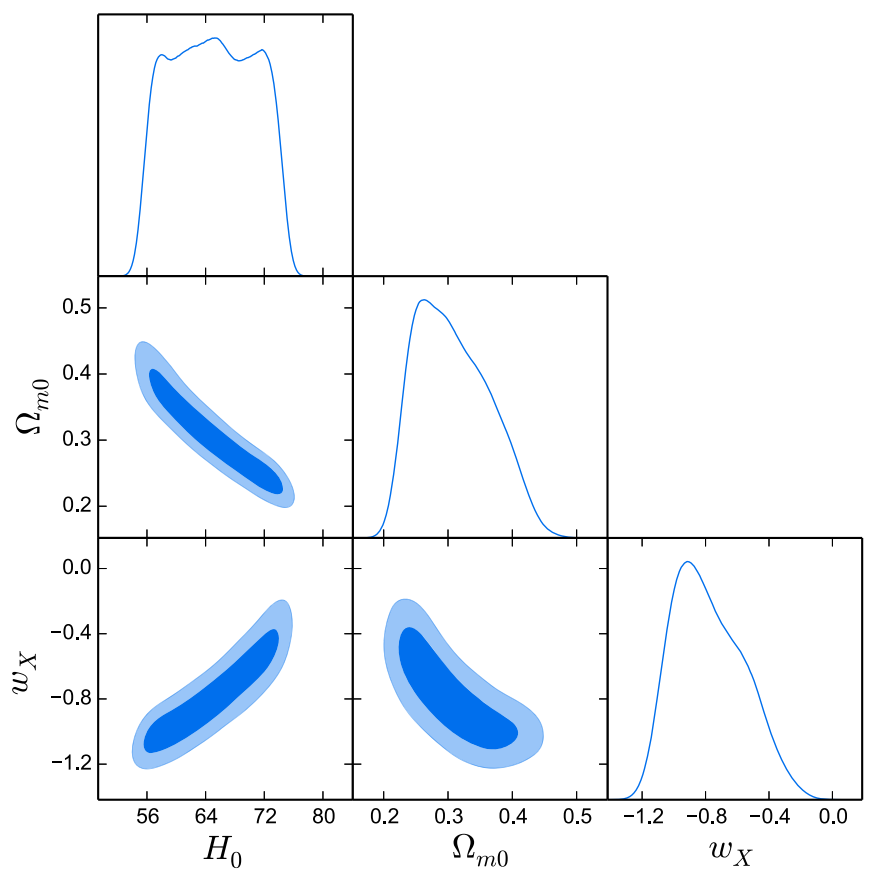

Figure 3. $1 \sigma$ and $2 \sigma$ confidence contours of the parameters of the spatially flat $\omega \mathrm{CDM}$ parameterization. Marginalized probability distributions of the individual parameters are also displayed.

function that results from marginalizing over all of the other parameters. The constraints listed in Table 2 are roughly in line with those now under discussion. The small reduced $\chi^{2}$ values that follow from the entries in the last line of the table are not unexpected given the results of Farooq et al. (2013a).

The $H_{0}$ values listed in Table 2 are in good accord with the lower recent values determined by using median statistics on Huchra's compilation and from CMB and BAO data, as well as with what is expected in the standard model of particle physics with only three light neutrino species and no additional "dark radiation."

There are two high-weight data subsets in our analysis: the cosmic chronometer data from Moresco et al. (2012) and the Ly $\alpha$ data from Busca et al. (2013). Since both of these results are based on relatively new approaches to measuring $H(z)$, it is informative to see an analysis of $H_{0}$ when one and then the other of these data sets is omitted from the analysis. When we drop the data of Moresco et al. (2012) from the compilation, we find $H_{0}=67.5_{-3.7-8.0}^{+3.7+8.0} \mathrm{~km} \mathrm{~s}^{-1} \mathrm{Mpc}^{-1}$, while by dropping the point of Busca et al. (2013) we obtain $H_{0}=$ $66.9_{-2.8-5.5}^{+2.8+5.3} \mathrm{~km} \mathrm{~s}^{-1} \mathrm{Mpc}^{-1}$. Comparing these with the full-data result $H_{0}=68.3_{-2.6-5.1}^{+2.7+5.2} \mathrm{~km} \mathrm{~s}^{-1} \mathrm{Mpc}^{-1}$, we observe a minor 


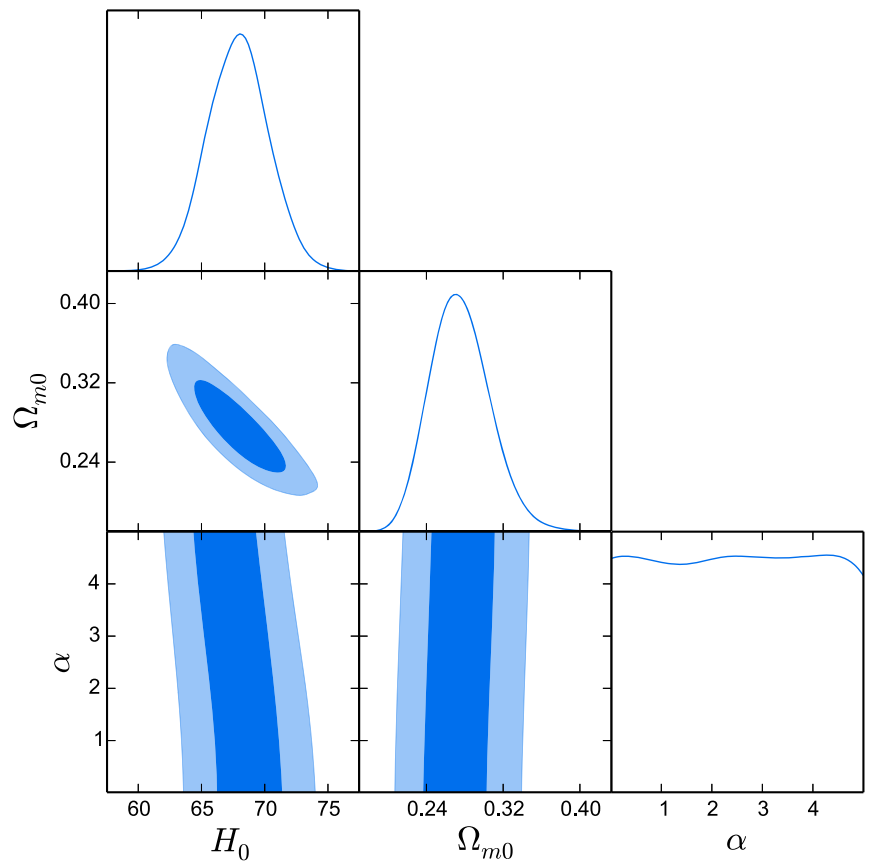

Figure 4. $1 \sigma$ and $2 \sigma$ confidence contours of the parameters of the spatially flat $\phi \mathrm{CDM}$ model. Marginalized probability distributions of the individual parameters are also displayed.

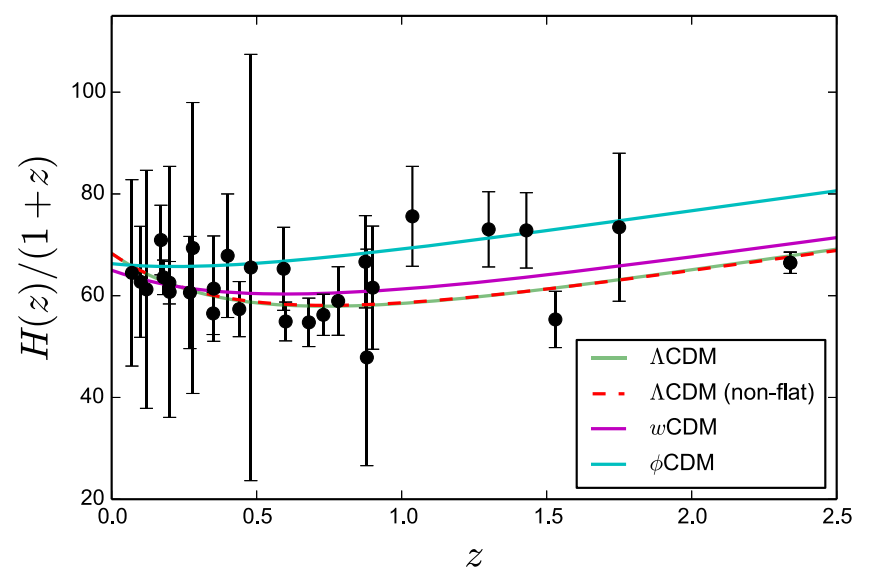

Figure 5. Best-fit model curves and the $28 H(z)$ data points.

shift in the central values and larger error bars when one or other data subset is omitted from the compilation.

\section{CONCLUSIONS}

We have used the $H(z)$ data tabulated in Farooq \& Ratra (2013b) as reproduced here in Table 1 to measure $H_{0}$. The $H_{0}$ values we find are more consistent with the lower values determined from the recent $\mathrm{CMB}$ and $\mathrm{BAO}$ data, as well as with the value found from a median statistics analysis of Huchra's compilation of $H_{0}$ measurements.

Y.C. was supported by the National Natural Science Foundation of China (Nos. 11133003 and 11573031), and the China Postdoctoral Science Foundation (No. 2015M571126). S.K. acknowledges support from SERB-DST project No. SR/FTP/PS-102/2011, DST FIST project No. SR/FST/MSI-090/2013(C), and the warm hospitality and research facilities provided by the Inter-University Center for Astronomy and Astrophysics (IUCAA), India where part of this work was carried out. B.R. was supported in part by DOE grant DEFG 03-99EP41093.

\section{REFERENCES}

Addison, G. E., Huang, Y., Watts, D. J., et al. 2016, ApJ, 818, 132 Ade, P. A. R., Aghanim, N., Arnaud, M., et al. 2015, A\&A, 594, A13 Alam, U., Bag, S., \& Sahni, V. 2016, arXiv:1605.04707

Andreon, S., \& Hurn, M. A. 2012, arXiv:1210.6232

Aubourg, E., Bailey, S., Bautista, J. E., et al. 2015, PhRvD, 92, 123516

Bernal, J. L., Verde, L., \& Riess, A. G. 2016, JCAP, 10, 019

Blake, C., Brough, S., Colless, M., et al. 2012, MNRAS, 425, 405

Busca, N. G., Delubac, T., Rich, J., et al. 2013, A\&A, 552, A96

Calabrese, E., Archidiacono, M., Melchiorri, A., \& Ratra, B. 2012, PhRvD, 86, 043520

Capozziello, S., Farooq, O., Luongo, O., \& Ratra, B. 2014, PhRvD, 90, 044016 Chen, G., Gott, J. R., \& Ratra, B. 2003, PASP, 115, 1269

Chen, G., \& Ratra, B. 2003, PASP, 115, 1143

Chen, G., \& Ratra, B. 2011a, PASP, 123, 1127

Chen, Y., Geng, C.-Q., Cao, S., Huang, Y.-M., \& Zhu, Z.-H. 2015, JCAP, 1502, 010

Chen, Y., \& Ratra, B. 2011b, PhLB, 703, 406

Chen, Y., Ratra, B., Biesiada, M., Li, S., \& Zhu, Z.-H. 2016, ApJ, 829, 61

Chuang, C.-H., \& Wang, Y. 2013, MNRAS, 435, 255

Crandall, S., Houston, S., \& Ratra, B. 2015, MPLA, 30, 25

Crandall, S., \& Ratra, B. 2014, PhLB, 732, 330

Crandall, S., \& Ratra, B. 2015, ApJ, 815, 87

Croft, R. A. C., \& Dailey, M. 2015, Quarterly Phys. Rev., 1, 1 (arXiv:1112.3108)

Ding, X., Biesiada, M., Cao, S., Li, Z., \& Zhu, Z.-H. 2015, ApJL, 803, L22

Di Valentino, E., Melchiorri, A., \& Silk, J. 2016, PhLB, 761, 242

Efstathiou, G. 2014, MNRAS, 440, 1138

Farooq, M. O. 2013, PhD thesis, Kansas State Univ. (arXiv:1309.3710)

Farooq, O., Crandall, S., \& Ratra, B. 2013a, PhLB, 726, 72

Farooq, O., Madiyar, F. R., Crandall, S., \& Ratra, B. 2016, arXiv:1607.03537

Farooq, O., Mania, D., \& Ratra, B. 2013b, ApJ, 764, 138

Farooq, O., Mania, D., \& Ratra, B. 2015, ApSS, 357, 11

Farooq, O., \& Ratra, B. 2013a, PhLB, 723, 1

Farooq, O., \& Ratra, B. 2013b, ApJL, 766, L7

Freedman, W. L., Madore, B. F., Gibson, B. K., et al. 2001, ApJ, 553, 47

Freedman, W. L., Madore, B. F., Scowcroft, V., et al. 2012, ApJ, 758, 24

Gott, J. R., Vogeley, M. S., Podariu, S., \& Ratra, B. 2001, ApJ, 549, 1

Guo, R.-Y., \& Zhang, X. 2016, EPJC, 76, 163

Hinshaw, G., Larson, D., Komatsu, E., et al. 2013, ApJS, 208, 19

Lewis, A., \& Bridle, S. 2002, PhRvD, 66, 103511

L'Huillier, B, \& Shafieloo, A. 2016, arXiv:1606.06832

Luković, V. V., D’Agostino, R., \& Vittorio, N. 2016, A\&A, 595, A109

Mamajek, E. E., \& Hillenbrand, L. A. 2008, ApJ, 687, 1264

Meng, X.-L., Wang, X., Li, S.-Y., \& Zhang, T.-J. 2015, arXiv:1507.02517

Moresco, M., Cimatti, A., Jimenez, R., et al. 2012, JCAP, 1208, 006

Moresco, M., Pozzetti, L., Cimatti, A., et al. 2016, JCAP, 1605, 014

Mukherjee, A. 2016, MNRAS, 460, 273

Peebles, P. J. E. 1984, ApJ, 284, 439

Peebles, P. J. E., \& Ratra, B. 1988, ApJL, 325, L17

Podariu, S., Souradeep, T., Gott, J. R., Ratra, B., \& Vogeley, M. S. 2001, ApJ, 559,9

Ratra, B., \& Peebles, P. J. E. 1988, PhRvD, 37, 3406

Riess, A. G., Macri, L., Casertano, S., et al. 2011, ApJ, 730, 119

Riess, A. G., Macri, L. M., Hoffmann, S. L., et al. 2016, ApJ, 826, 56

Ross, A. J., Samushia, L., Howlett, C., et al. 2015, MNRAS, 449, 835

Samushia, L. 2009, PhD thesis, Kansas State Univ. (arXiv:0908.4597)

Samushia, L., Chen, G., \& Ratra, B. 2007, arXiv:0706.1963

Samushia, L., \& Ratra, B. 2006, ApJL, 650, L5

Sievers, J. L., Hlozek, R. A., Nolta, M. R., et al. 2013, JCAP, 1310, 060

Simon, J., Verde, L., \& Jimenez, R. 2005, PhRvD, 71, 123001

Solà, J., Gómez-Valent, A., \& de Cruz Pérez, J. 2016, arXiv:1602.02103

Stern, D., Jimenez, R., Verde, L., Kamionkowski, M., \& Stanford, S. A. 2010, JCAP, 1002, 008

Verde, L., Protopapas, P., \& Jimenez, R. 2014, PDU, 5-6, 307

Zhang, C., Zhang, H., Yuan, S., et al. 2014, RAA, 14, 1221

Zheng, X., Ding, X., Biesiada, M., Cao, S., \& Zhu, Z.-H. 2016, ApJ, 825, 17 